\title{
EDITORIAL
}

\section{Physicians and erectile dysfunction}

\author{
Richard W Casey MD FRCSC
}

E rectile dysfunction (ED) is a complex psychosocial health issue that requires a multidisciplinary approach to fully understand and treat the patient and his partner. ED is a relatively simple mechanical dysfunction that is treated by restoring penile rigidity [confirm]. You decide.

ED was an orphan disease until the introduction of intracorporal injection (ICI) therapy and was considered largely to be a symptom of a more complex psychological problem. With the availability of a means to restore penile rigidity [confirm], urologists saw their practices expand, and physicians' impressions of the problem changed. With treatment [confirm] no longer being the domain of the 'sexologist' or psychologist, patients seemed happier with a simple solution that provided them with a 'quick fix' and that did not require $12,1 \mathrm{~h}$ sessions. The excitement that ICI therapy generated lead to the establishment of ED clinics and 'injection mills'. In the poorly trained physician's mind, ICI therapy became the answer to all penile problems, from premature ejaculation to Peyronie's disease. ED clinics, usually organized by urologists at teaching centres, provided the patient with counselling and partner support when necessary. Those of us who worked in these clinics began to notice that most patients were not interested in exploring their 'sexuality' and that their partners were not interested in attending the clinic with them. The partners' attitude of 'Come home when you can get an erection' was relayed to us by our patients.

The introduction of sildenafil has changed the landscape again. Family physicians are now actively involved in treat- ing ED. Physicians who have a relationship with the patients and their families are probably best suited to provide ED solutions. Complex medical patients, treatment failures and patients requiring surgical intervention, however, will require the expertise of urologists. The article about ED and counselling issues by Lee et al (pages $\mathbf{x x x}-\mathbf{x x x}$ ) in this issue suggests that what most urologists have felt for years may be true. Patients and their partners, for the most part, are able to put ED in perspective and accept a simple medical or surgical solution. Physicians can identify those patients who require counselling or psychiatric assessment, and these patients need not be part of a routine treatment program. ED clinics can deliver education and support to patients, but, most importantly, they can develop as Centres of Excellence evaluating new treatments and providing training to physicians interested in developing this aspect of their practices. Injection mills will eventually close as patients find their physicians more willing to discuss sexual problems as a component of their overall care.

Now that we have made good progress with ED, it is time to turn our attention to female sexual dysfunction (FSD). In this issue, Dr Gladu (pages $\mathbf{x x x}-\mathbf{x x x}$ ) outlines an approach to the investigation and treatment of this disorder, directed by the family physician. Is FSD a complex psychosocial health issue that requires a multidisciplinary approach to fully understand and treat the patient and her partner, or is it a relatively simple mechanical dysfunction that is treated by providing the patient with adequate genital blood flow? You decide.

The Male Health Centres, Oakville, Ontario

Correspondence: Dr Richard W Casey, 407-1235 Trafalgar Road North, Oakville, Ontario L6H 3P1. Telephone 905-338-3130, fax 905-338-3150, e-mail drcasey@malehealth.com 